\title{
Genome sequence of Corynebacterium Amycolatum ICIS 99 Isolated From Human Vagina Reveals Safety and Beneficial Properties
}

\section{Irina Gladysheva ( $\sim$ GladishevalV@yandex.ru )}

Research Institute for Cellular and Intracellular Symbiosis, https://orcid.org/0000-0001-6231-7028

\section{Yuriy Khlopko}

Institute for Cellular and Intracellular Symbiosis UB RAS: Institut kletocnogo i vnutrikletocnogo simbioza UrO RAN

\section{Vladimir Kataev}

Institute for Cellular and Intracellular Symbiosis UB RAS: Institut kletocnogo i vnutrikletocnogo simbioza UrO RAN

\section{Sergey Cherkasov}

Institute for Cellular and Intracellular Symbiosis UB RAS: Institut kletocnogo i vnutrikletocnogo simbioza UrO RAN

\section{Research Article}

Keywords: Corynebacterium Amycolatum, Human Vagina, bacteriocins

Posted Date: December 23rd, 2021

DOI: https://doi.org/10.21203/rs.3.rs-1186343/v1

License: (9) This work is licensed under a Creative Commons Attribution 4.0 International License. Read Full License

Version of Record: A version of this preprint was published at Archives of Microbiology on March 29th, 2022. See the published version at https://doi.org/10.1007/s00203-022-02852-7. 


\section{Abstract}

Corynebacterium amycolatum ICIS 99 was isolated from vaginal smears of healthy women and showed promising results in antimicrobial screenings. Here, we report the draft genome sequence of this strain and analyze its main features to assess its safety and useful properties. The genome is 2,532,503 bp long and contains 2,186 CDSs with an average G+C content of $59.0 \%$. Analyses of the ICIS 99 genome revealed the absence of true virulence genes. The genome contains genes involved in the synthesis of secondary metabolites and bacteriocins of the class sactipeptide. In the genome of ICIS 99, we identified a large number of genes responsible for adaptation and survival in the vaginal environment, including acid and oxidative stress resistance genes. The genomic information of ICIS 99 provides a basis for understanding the safety and useful properties of ICIS 99 and for considering it as a potential probiotic strain. However, further research is needed to test its probiotic efficacy in vivo. The whole genome shotgun project has been deposited at DDBJ/ENA/GenBank under the accession number JAIUSU000000000.

\section{Introduction}

The genus Corynebacterium was described in 1896 by Lehmann \& Neumann and includes straight or slightly curved rod-shaped, gram-positive, catalase-positive, non-spore-forming, nonmotile bacteria that belong to the family Corynebacteriaceae of the phylum Actinobacteria (Lehmann and Neumann 2016). Currently, this genus contains 120 valid species (www.bacterio.net/corynebacterium.html). Corynebacterium are widespread in the environment and can be isolated from soil, water, and plant material. Certain species of corynebacteria are of great importance in industry and agriculture (Ikeda and Takeno 2012). Some Corynebacterium spp. are well-known pathogens of humans or animals; other species are part of the normal microflora, although they may occasionally cause infections in humans or can be transmitted to humans by zoonotic contact (Tauch and Sandbote 2014).

Corynebacterium amycolatum is a typical bacterium of the flora on human skin and mucous membranes. It was first isolated by Collins and Burton from clinical specimens in 1988 (Collins et al. 1988). A distinctive feature of this species compared to other corynebacteria is the absence of typical mycolic acids in the cell membrane. According to the 16S rRNA alignment analysis of different Corynebacterium species, C. amycolatum is only distantly related to other corynebacteria (Pascual et al.1995).

In the literature, studies on $C$. amycolatum are limited, and this species is described as a causative agent of sepsis, endocarditis, meningitis, septic arthritis, and urinary tract infections (Reddy et al. 2012). However, the similarity of nondiphtheria corynebacteria often calls into question the identification of isolates from clinical specimens (Esteban et al. 1999).

In this study, we report the draft genome sequence of $C$. amycolatum ICIS 99, which was isolated from vaginal smears of healthy women during our pilot study aimed at investigating the biological properties of isolates of Corynebacterium spp. In a previous study, the strain was identified as Corynebacterium 
xerosis by biochemical tests of the Api Coryne system (Gladysheva et al.2017). Cell-free supernatants (CFSs) from this strain showed promising results in antimicrobial screenings. A preliminary safety screening revealed that ICIS 99 did not show hemolytic, DNase, or gelatinase activities (data unpublished).

Therefore, the present study was undertaken to study the genome of this strain to assess its safety and useful properties.

\section{Materials And Methods}

\section{Bacterial strain and genomic DNA extraction}

C. amycolatum ICIS 99 was isolated from the vaginal contents of healthy women and is part of the Collection of Microorganisms of the Institute for Cellular and Intracellular Symbiosis UrB RAS (Orenburg, Russia). The strains were grown in Luria-Bertani (LB) Miller broth (Becton Dickinson, USA) at $37^{\circ} \mathrm{C}$ for 24 $\mathrm{h}$ and subcultured once in the same medium at $37^{\circ} \mathrm{C}$ for $24 \mathrm{~h}$ before use. The strain morphologies were observed by scanning electron microscopy (SEM) (Fig. 1).

Genomic DNA was extracted using a Quick DNA Fungal/Bacterial Kit (Zymo Research, USA). The quality of the extracted DNA was assessed according to the A260/280 ratio using a Nanodrop 8000 (Thermo Fisher Scientific, USA), and electrophoresis was performed in a 1\% agarose gel. DNA concentration was quantified by using a Qubit 4 Fluorometer and a dsDNA High Sensitivity Assay Kit (Life Technologies, USA).

\section{Genome sequencing, assembly and annotation}

The DNA library for whole-genome sequencing was prepared using a NEBNext ${ }^{\circledR}$ Ultra ${ }^{\mathrm{TM}}$ II FS DNA Library Prep Kit for Illumina ${ }^{\circledR}$ (New England BioLabs, USA). The library was validated using capillary electrophoresis on a QIAxcel Advanced System (Qiagen, Germany) using the QIAxcel DNA High Resolution Kit and normalized using qPCR on the CFX Connect Real-Time PCR System (Bio Rad, USA). Paired-end sequencing ( $2 \times 250 \mathrm{bp}$ ) was carried out on a MiSeq platform (Illumina, USA) using Reagent Kit v.2 (Illumina, USA) in the Center of Shared Scientific Equipment "Persistence of microorganisms" at the Institute for Cellular and Intracellular Symbiosis UrB RAS.

The paired-end reads were filtered and trimmed with the Trimmomatic program (Bolger et al.2014). De novo genome assembly was performed using the Unicycler v. 0.4.9b genome assembler (Wick et al.2017). The contigs were analyzed for gene prediction using the NCBI Prokaryotic Genome Annotation Pipeline (PGPA) and rapid annotation using subsystem technology (RAST) (Aziz et al. 2008). RAST data visualization was performed using GraphPad Prism version 9.2.0 and GraphPad Software (La Jolla, CA, USA) (www.graphpad.com). The interactive visualization of the genome of Corynebacterium amycolatum ICIS 99 was performed with GView (Petkau et al. 2010). 
Tentative taxonomic identification was carried out using the BLASTn 2.12.0+ program, according to the rRNA/ITS databases Targeted Loci Project. Summary results are presented in Supplementary Table 1.

Average nucleotide identity (ANI) values were calculated with the Orthologous Average Nucleotide Identity tool (Lee et al. 2016). The following were used for comparison: the complete reference genome of $C$. amycolatum FDAARGOS 1108 (CP068168); five draft genomes of $C$. amycolatum strains currently available from the NCBI database, including SK46 (WGS project: ABZU01), UMB0042 (PKHS01), ICIS 5 (SSOR01), ICIS 9 (MTPT01) and ICIS 53 (MIFV01); and complete reference genomes of the species Corynebacterium xerosis (CP032788), Corynebacterium lactis (CP006841), and Corynebacterium jeikeium (NZ_LS483459).

The bioinformatic tools antiSMASH 5.0 (Blin et al. 2021), NaPDoS (Ziemert et al. 2012) and BAGEL4 (Heel et al. 2018) were used to determine potential clusters of secondary metabolites with antimicrobial activity. Antibiotic resistance genes in the genomes were predicted using the RGI (Resistance Gene Identifier) tool (Alcock et al. 2020). The presence of putative virulence genes in the genomes was investigated using the Virulence Factor of Bacterial Pathogens Database (VFDB) (Liu et al. 2019). The CRISPR regions were identified with the CRISPR online detection tool CRISPR finder (https://crisprcas.i2bc.paris-saclay.fr/CrisprCasFinder/Index) (Couvin et al. 2018).

\section{Nucleotide sequence accession number}

This whole genome shotgun project has been deposited at DDBJ/ENA/GenBank under the accession JAIUSU000000000. The version described in this paper is version JAIUSU010000000.

\section{Results And Discussion}

\section{General genomic features and genome-based phylogeny of ICIS 99}

The genome characteristics of strain ICIS 99 are presented in Table 2 and Fig. 2. The draft genome of ICIS 99 was 2,532,503 bp long with an N50 length of 167,044 bp, an L50 of 6, and a G+C content of $59.0 \%$. The final assembled genome consisted of 41 contigs. Genome annotation was performed using the National Center for Biotechnology Information (NCBI) Prokaryotic Genome Annotation Pipeline (PGAP) (http://www.ncbi.nlm.nih.gov/genome/annotation_prok), and 2,186 coding sequences, including 2,156 proteins (CDSs), 30 pseudogenes, 3 complete rRNAs (5S, 16S, and 23S) and 54 tRNAs, were identified.

The RAST subsystem annotated the coding proteins into 22 functional categories based on their COG classifications (Fig. 3). Most genes belonged to the categories Protein Metabolism (17.75\%), Amino Acids and Derivatives (16.43\%), Carbohydrates (12.78\%), Cofactors, Vitamins, Prosthetic Groups, and Pigments (9.63\%), Nucleosides and Nucleotides (5.78\%), Respiration (4.87\%) and Fatty Acids, Lipids, and Isoprenoids (4.77\%). 
ANI and OrthoANI analyses made it possible to definitively determine that ISIS 99 was a $C$.

amycolatum strain. For comparison, the following genomes were used: the complete reference genome $C$. amycolatum FDAARGOS 1108 (CP068168); five draft genomes of $C$. amycolatum strains currently available from the NCBI database, including SK46 (WGS project: ABZU01), UMB0042 (PKHS01), ICIS 5 (SSOR01), ICIS 9 (MTPT01) and ICIS 53 (MIFV01); and complete reference genomes of Corynebacterium xerosis (CP032788), Corynebacterium lactis (CP006841), and Corynebacterium jeikeium (NZ_LS483459).

The ANI value between ICIS 99 and seven strains of C. amycolatum ranged from 94.83 to $97.94 \%$ (Fig. 4), and the OrthoANI value ranged from 94.85 to $97.94 \%$ (Supplementary Fig. 1). Relative to other Corynebacterium strains (C. xerosis (CP032788), C. lactis (CP006841), and C. jeikeium (NZ_LS483459), the ANI values ranged from 73.87 to $76.42 \%$ (Fig. 4), and the OrthoANI values ranged from 71.73 to 77.05\% (Supplementary Fig. 1).

\section{Unique genomic characteristics of ICIS 99}

To assess the safety of this strain, we examined the genome for the presence of genes related to antibiotic resistance and virulence. The RGI tool was used to predict antibiotic resistance genes in ICIS 99. In the genome of ICIS 99, only one gene was found to code for resistance to the antibiotic chloramphenicol.

VFDB software was used to predict virulence factors in ICIS 99. VFDB predicted 18 virulence factors in ICIS 99 (Supplementary Table 2). These virulence factors can be classified into ten categories: adherence, iron uptake, regulation, amino acid and purine metabolism, antiphagocytosis, cell surface components, immune evasion, lipid and fatty acid metabolism, protease and secretion system. However, none of the genes identified were true virulence genes. These genes encode only proteins responsible for the structural and functional characteristics of microorganisms of the genus Corynebacteria or 'niche factors' (Swierczynski and Ton-That 2006; Wennerhold et al. 2006; Tauch et al. 2015; Baumgart et al. 2016; Bush 2018). True virulence genes, such as toxin-related genes (diphtheria toxin and phospholipase $D$ ) and hemolysin-related genes characteristic of well-known pathogenic corynebacteria, were not identified.

The previously described antibacterial activity of CFSs from ICIS 99 suggests that this strain contains genes responsible for the synthesis of secondary metabolites and bacteriocins. The presence of secondary metabolic gene clusters was examined by the antiSMASH 5.0 platform (Blin et al. 2019) and NaPDoS (Ziemert et al. 2012). AntiSMASH 5.0 revealed 4 putative biosynthetic gene clusters, which were predicted to be related to the biosynthesis of different types of secondary metabolites, including terpene, T3pks (type III polyketide synthases), NAPAA and NRPS (nonribosomal peptide) (Supplementary Table 3). Two gene clusters showed low similarity $(\leq 10 \%)$ with the most similar clusters, of which T3pks gene clusters were associated with the biosynthesis of merochlorin A-D-like compounds and the Nrps gene cluster was associated with the biosynthesis of phthoxazolin-like compounds. The other two gene clusters did not resemble any registered gene clusters. NaPDoS revealed one putative gene cluster, T2pks (type II polyketide synthase), that was involved in the biosynthesis of actinorhodin (similarity $27 \%$ ). The 
existence of clusters of secondary metabolic genes indicates that the ICIS 99 strain can be a producer of new natural bioactive products.

To search for genes involved in the synthesis of potential bacteriocins, we used BAGEL4. The genome of strain ICIS 99 had one area of interest (AOI) that included the class sactipeptide (Fig. 5 and Supplementary Table 4). Sactipeptides are a new class of ribosomally synthesized and posttranslationally modified peptides (RiPPs) and are a growing class of natural products that have garnered substantial attention because of their structural diversity and biological activities. Sactipeptides exhibit narrow-spectrum antibiotic activity against Clostridia and other drug-resistant bacteria (Balty et al. 2019).

In the genome of ICIS 99, we identified a large number of genes coding for proteins involved in the stress response. These stresses include $\mathrm{pH}$, osmotic pressure, nitrogen stress and oxidative stress. The presence of these genes determines the ability of ICIS 99 to adapt to and colonize the vaginal biotope of healthy women, which is a fairly aggressive environment for most pathogenic and opportunistic microorganisms. The detailed analysis of genes coding for proteins involved in stress response in the genome of ICIS 99 is shown in Supplementary Table 5.

The genome of ICIS 99 was analyzed for the occurrence and diversity of CRISPR-Cas systems. The identification of CRISPR loci suggests the presence of a prokaryotic immune system, which confers resistance to foreign genetic elements and provides a form of acquired immunity (Amitai and Sorek 2016). The ICIS 99 genome contained three CRISPR loci (CRISPR1-CRISPR3) (Supplementary Table 6). The detected CRISPR1/CRISPR-associated (Cas) system was type IE (Cas5, Cas7, Cse2, Cas6, Cas3, Cas1, and Cas2).

In conclusion, the genomic information for ICIS 99 provides the basis for understanding the safety and useful properties of ICIS 99 and for considering its use as a potential probiotic strain. However, further research is needed to test its probiotic efficacy in vivo.

Table 1 General genome features of Corynebacterium amycolatum ICIS 99 


\begin{tabular}{|ll|}
\hline \multicolumn{1}{|c|}{ Feature } & Value \\
\hline Coverage & $375 x$ \\
Number of contigs & 41 \\
\hline N50 & 167,044 \\
\hline L50 & 6 \\
\hline Depth of coverage & 375 \\
\hline Genome size (b.p.) & $2,532,503$ \\
\hline GC contents (\%) & 59,0 \\
\hline Genes (total) & 2,246 \\
\hline CDSs(total) & 2,186 \\
\hline Genes (coding) & 2,156 \\
\hline CDSs (with protein) & 2,156 \\
\hline rRNAs (5S, 16S, 23S) & $3(1,1,1)$ \\
\hline complete rRNAs & $1,1,1$ (5S, 16S, 23S) \\
\hline tRNAs & 54 \\
\hline Pseudo Genes (total) & 30 \\
\hline BioProject & PRJNA339674 \\
\hline BioSample & SAMN21545782 \\
\hline GenBank accession no & JAlUSU000000000 \\
\hline
\end{tabular}

\section{Declarations}

\section{Availability of data and materials}

All data generated or analyzed during this study are included in this published article and its Supplementary Information files. Additional data are available from the corresponding author upon request.

\section{Acknowledgments}

The authors are grateful to Marina Ignatenko, employee of the Center for Revealing and Support of Gifted Children "Gagarin", Orenburg region, Russia, for conducting scanning electron microscopy of the samples.

\section{Conflict of interest}


The authors have no financial conflicts of interest to declare.

\section{Author Contribution}

Irina Gladysheva designed a study. Vladimir Kataev carried out the experimental design. Yuriy Khlopko carried out bioinformatic analysis. Irina Gladysheva and Sergey Cherkasov analyzed data and prepared the manuscript. All authors have read and approved the final manuscript.

\section{References}

Alcock BP, Raphenya AR, Lau TTY, et al (2020) CARD 2020: antibiotic resistome surveillance with the comprehensive antibiotic resistance database. Nucleic Acids Research 48:D517-D525. https://doi.org/10.1093/NAR/GKZ935

Amitai G, Sorek R (2016) CRISPR-Cas adaptation: insights into the mechanism of action. Nature Reviews Microbiology 2016 14:2 14:67-76. https://doi.org/10.1038/nrmicro.2015.14

Aziz RK, Bartels D, Best A, et al (2008) The RAST Server: Rapid annotations using subsystems technology. BMC Genomics 9:1-15. https://doi.org/10.1186/1471-2164-9-75/TABLES/3

Balty C, Guillot A, Fradale L, et al (2019) Ruminococcin C, an anti-clostridial sactipeptide produced by a prominent member of the human microbiota Ruminococcus gnavus. The Journal of biological chemistry 294:14512-14525. https://doi.org/10.1074/JBC.RA119.009416

Baumgart M, Schubert K, Bramkamp M, Frunzke J (2016) Impact of LytRCpsA-Psr proteins on cell wall biosynthesis in Corynebacterium glutamicum. Journal of Bacteriology 198:3045-3059. https://doi.org/10.1128/JB.00406-16/SUPPL_FILE/ZJB999094215S01.PDF

Blin K, Shaw S, Kloosterman AM, et al (2021) antiSMASH 6.0: improving cluster detection and comparison capabilities. Nucleic Acids Research 49:W29-W35. https://doi.org/10.1093/NAR/GKAB335

Bolger AM, Lohse M, Usadel B (2014) Trimmomatic: a flexible trimmer for Illumina sequence data. Bioinformatics 30:2114-2120. https://doi.org/10.1093/BIOINFORMATICS/BTU170

Bush MJ (2018) The actinobacterial WhiB-like (Wbl) family of transcription factors. Molecular Microbiology 110:663-676. https://doi.org/10.1111/MMI.14117

Collins MD, Burton RA, Jones D (1988) Corynebacterium amycolatum sp. nov. a new mycolic acid-less Corynebacterium species from human skin. FEMS Microbiology Letters 49:349-352. https://doi.org/10.1111/j.1574-6968.1988.tb02755.x

Couvin D, Bernheim A, Toffano-Nioche C, et al (2018) CRISPRCasFinder, an update of CRISRFinder, includes a portable version, enhanced performance and integrates search for Cas proteins. Nucleic Acids Research 46:W246-W251. https://doi.org/10.1093/NAR/GKY425 
Esteban J, Nieto E, Calvo R, et al (1999) Microbiological characterization and clinical significance of Corynebacterium amycolatum strains. European Journal of Clinical Microbiology and Infectious Diseases 18:518-521. https://doi.org/10.1007/S100960050336

Gladysheva IV., Cherkasov SV., KhlopkoY (2017) Antibacterial activities of metabolites from Corynebacterium spp. Strains isolated from the reproductive Tract of a healthy woman against human pathogenic bacteria. International Journal of Pharma and Bio Sciences 8:.

https://doi.org/10.22376/IJPBS.2017.8.3.B549-556

Ikeda M, Takeno S (2013) Amino Acid Production by Corynebacterium glutamicum. 107-147. https://doi.org/10.1007/978-3-642-29857-8_4

Lee I, Kim YO, Park SC, Chun J (2016) OrthoANI: An improved algorithm and software for calculating average nucleotide identity. International Journal of Systematic and Evolutionary Microbiology 66:11001103. https://doi.org/10.1099/IJSEM.0.000760/CITE/REFWORKS

Lehmann Karl Bernhard (2016) Atlas und Grundriss der Bakteriologie und Lehrbuch der speziellen bakteriologischen Diagnostik /. Atlas und Grundriss der Bakteriologie und Lehrbuch der speziellen bakteriologischen Diagnostik /. https://doi.org/10.5962/BHL.TITLE.117384

Liu B, Zheng D, Jin Q, et al (2019) VFDB 2019: a comparative pathogenomic platform with an interactive web interface. Nucleic Acids Research 47:D687-D692. https://doi.org/10.1093/NAR/GKY1080

Pascual C, Lawson PA, Farrow JAE, et al (1995) Phylogenetic analysis of the genus Corynebacterium based on $16 \mathrm{~S}$ rRNA gene sequences. International journal of systematic bacteriology 45:724728. https://doi.org/10.1099/00207713-45-4-724

Petkau A, Stuart-Edwards M, Stothard P, van Domselaar G (2010) Interactive microbial genome visualization with GView. Bioinformatics 26:3125-3126. https://doi.org/10.1093/bioinformatics/btq588

Reddy BS, Chaudhury A, Kalawat U, et al (2012) Isolation, speciation, and antibiogram of clinically relevant non-diphtherial Corynebacteria (Diphtheroids). Indian journal of medical microbiology 30:52-57. https://doi.org/10.4103/0255-0857.93033

Swierczynski A, Ton-That H (2006) Type III Pilus of Corynebacteria: Pilus Length Is Determined by the Level of Its Major Pilin Subunit. Journal of Bacteriology 188:6318. https://doi.org/10.1128/JB.00606-06

Tauch A, Burkovski A (2015) Molecular armory or niche factors: virulence determinants of Corynebacterium species. FEMS microbiology letters 362:. https://doi.org/10.1093/FEMSLE/FNV185

Tauch A, Sandbote J (2014) The Family Corynebacteriaceae. The Prokaryotes: Actinobacteria 239-277. https://doi.org/10.1007/978-3-642-30138-4_187 
Van Heel AJ, De Jong A, Song C, et al (2018) BAGEL4: a user-friendly web server to thoroughly mine RiPPs and bacteriocins. Nucleic Acids Research 46:W278-W281. https://doi.org/10.1093/NAR/GKY383

Wennerhold J, Bott M (2006) The DtxR regulon of Corynebacterium glutamicum. Journal of Bacteriology 188:2907-2918. https://doi.org/10.1128/JB.188.8.2907-

2918.2006/SUPPL_FILE/SUPPLEMENTAL_MATERIAL_WENNERHOLD_AND_BOTT.PDF

Wick RR, Judd LM, Gorrie CL, Holt KE (2017) Unicycler: Resolving bacterial genome assemblies from short and long sequencing reads. PLOS Computational Biology 13:e1005595.

https://doi.org/10.1371/JOURNAL.PCBI.1005595

Ziemert N, Podell S, Penn K, et al (2012) The Natural Product Domain Seeker NaPDoS: A Phylogeny Based Bioinformatic Tool to Classify Secondary Metabolite Gene Diversity. PLOS ONE 7:e34064. https://doi.org/10.1371/JOURNAL.PONE.0034064

\section{Figures}




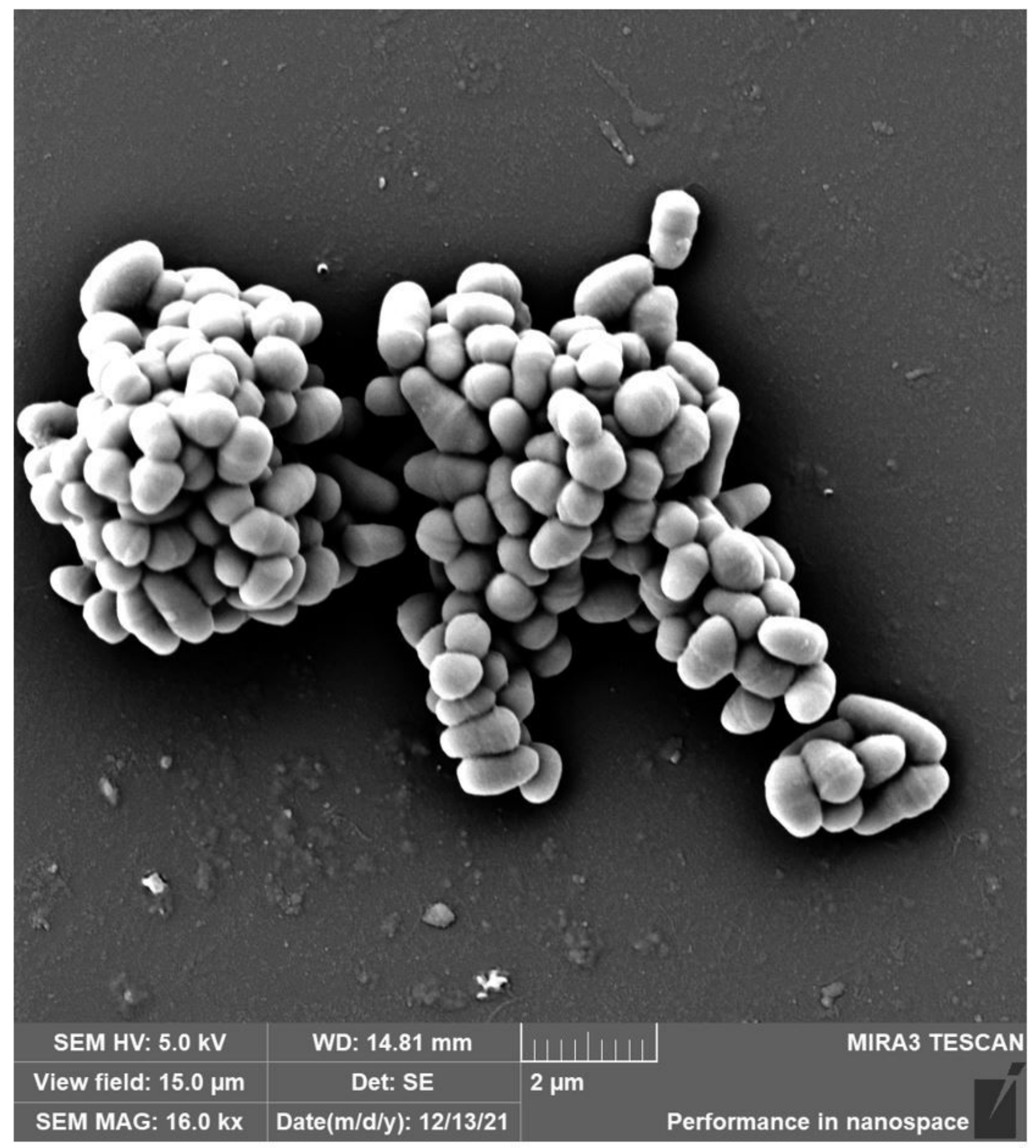

\section{Figure 1}

Scanning electron microscope of Corynebacterium amycolatum ICIS 99 


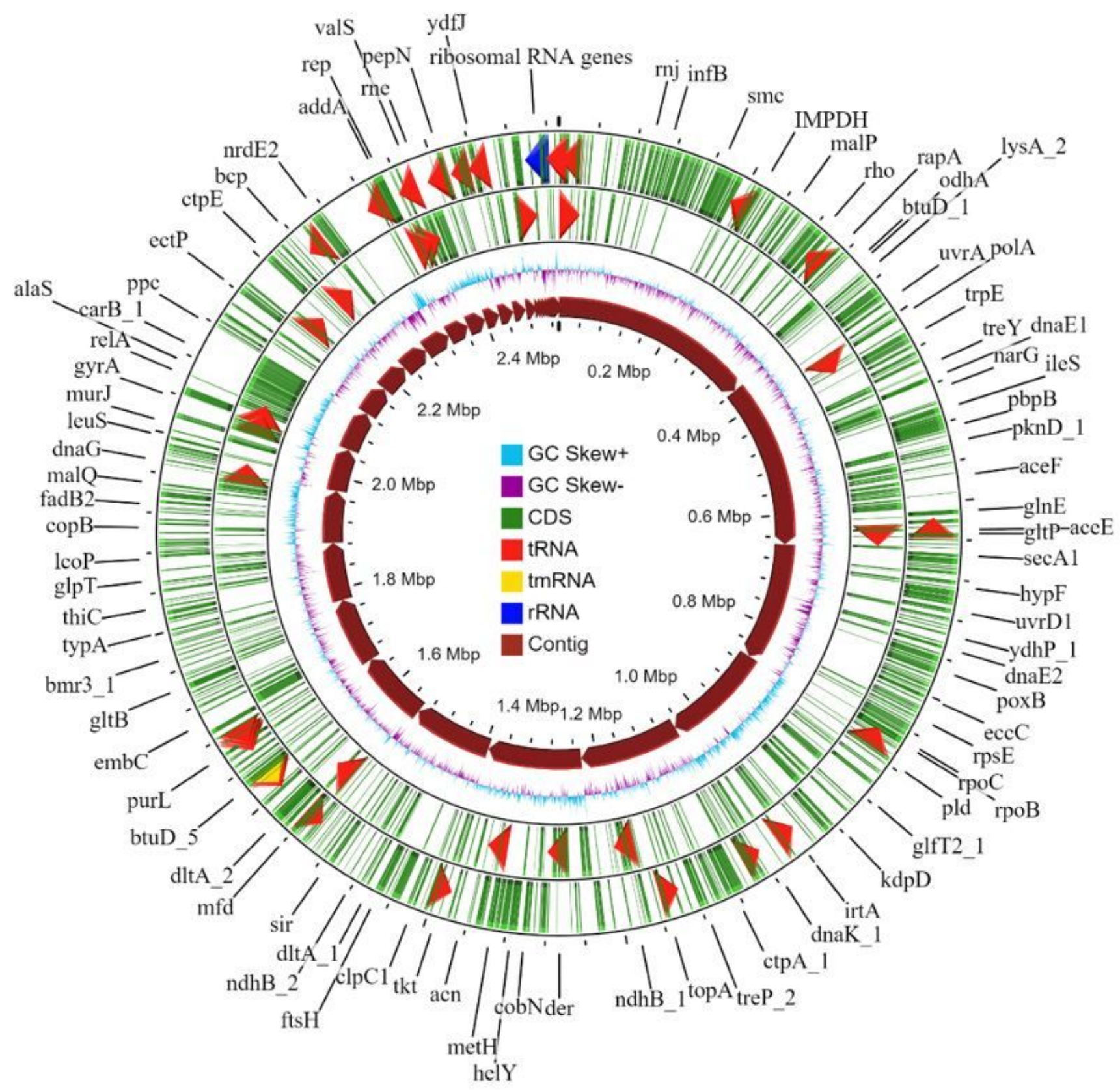

Figure 2

Circular map of the genome of Corynebacterium amycolatum ICIS 99. Each ring represents the loci of genes that are labeled outside the outermost ring: (from outermost to innermost) forward coding sequences (green); reverse coding sequences (green); contigs (dark red); GC skew +/- (blue/violet); genome size (black). Triangles within rings: rRNA (dark blue); tRNA (red); tmRNA (yellow). 


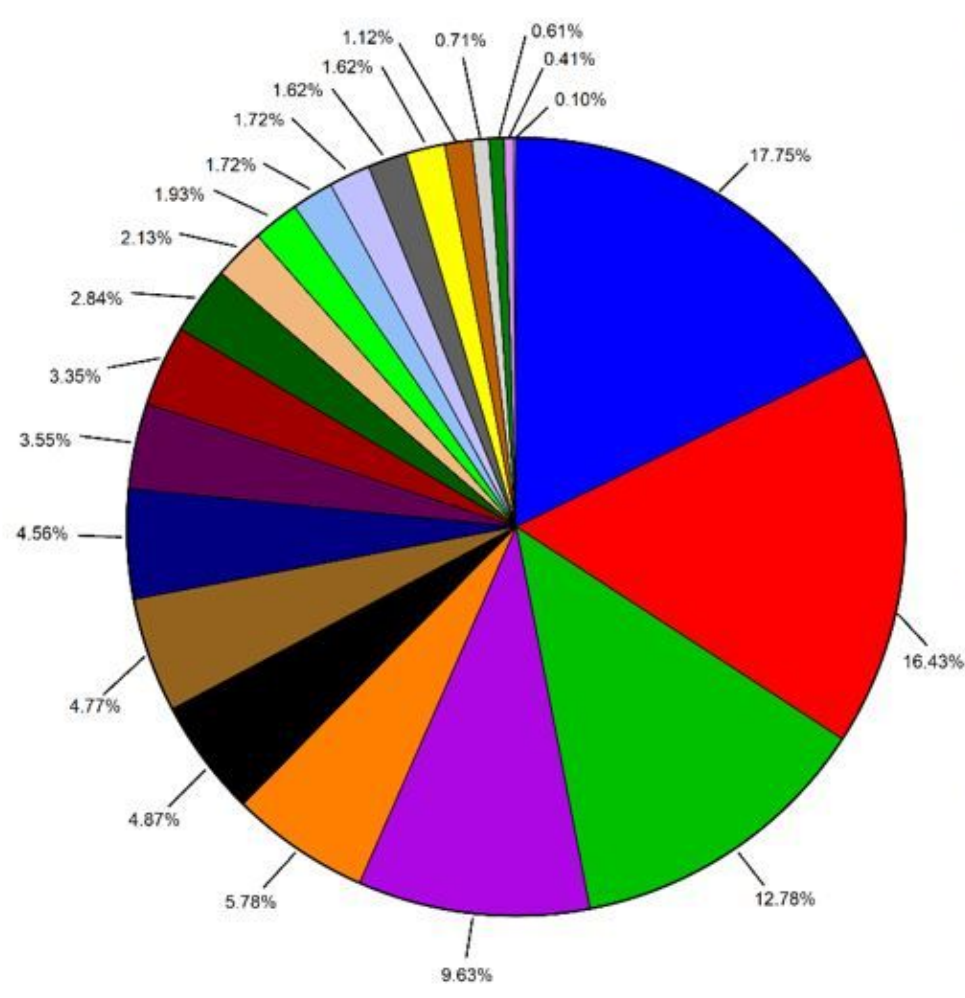

- $17.75 \%$ Protein Metabolism

16.43\% Amino Acids and Derivatives

12.78\% Carbohydrates

9.63\% Cofactors, Vitamins, Prosthetic Groups, Pigments

$\square .78 \%$ Nucleosides and Nucleotides

- $4.87 \%$ Respiration

$\square \quad 4.77 \%$ Fatty Acids, Lipids, and Isoprenoids

- $4.56 \%$ DNA Metabolism

3.55\% Membrane Transport

$3.35 \%$ RNA Metabolism

$2.84 \%$ Virulence, Disease and Defense

$\square \quad 2.13 \%$ Cell Wall and Capsule

1.93\% Phosphorus Metabolism

$\square 1.72 \%$ Miscellaneous

$\square 1.72 \%$ Nitrogen Metabolism

$\square 1.62 \%$ Regulation and Cell signaling

$\square 1.62 \%$ Stress Response

- $1.12 \%$ Iron acquisition and metabolism

$\square .71 \%$ Potassium metabolism

$0.61 \%$ Metabolism of Aromatic Compounds

$\square \quad 0.41 \%$ Sulfur Metabolism

$\square \quad 0.10 \%$ Dormancy and Sporulation

\section{Figure 3}

Subsystem category distribution of major protein-coding genes of Corynebacterium amycolatum ICIS 99 as annotated by the RAST annotation server. The bar chart shows the subsystem coverage in percentage. The pie chart shows percentage distribution of the 22 most abundant subsystem categories. 


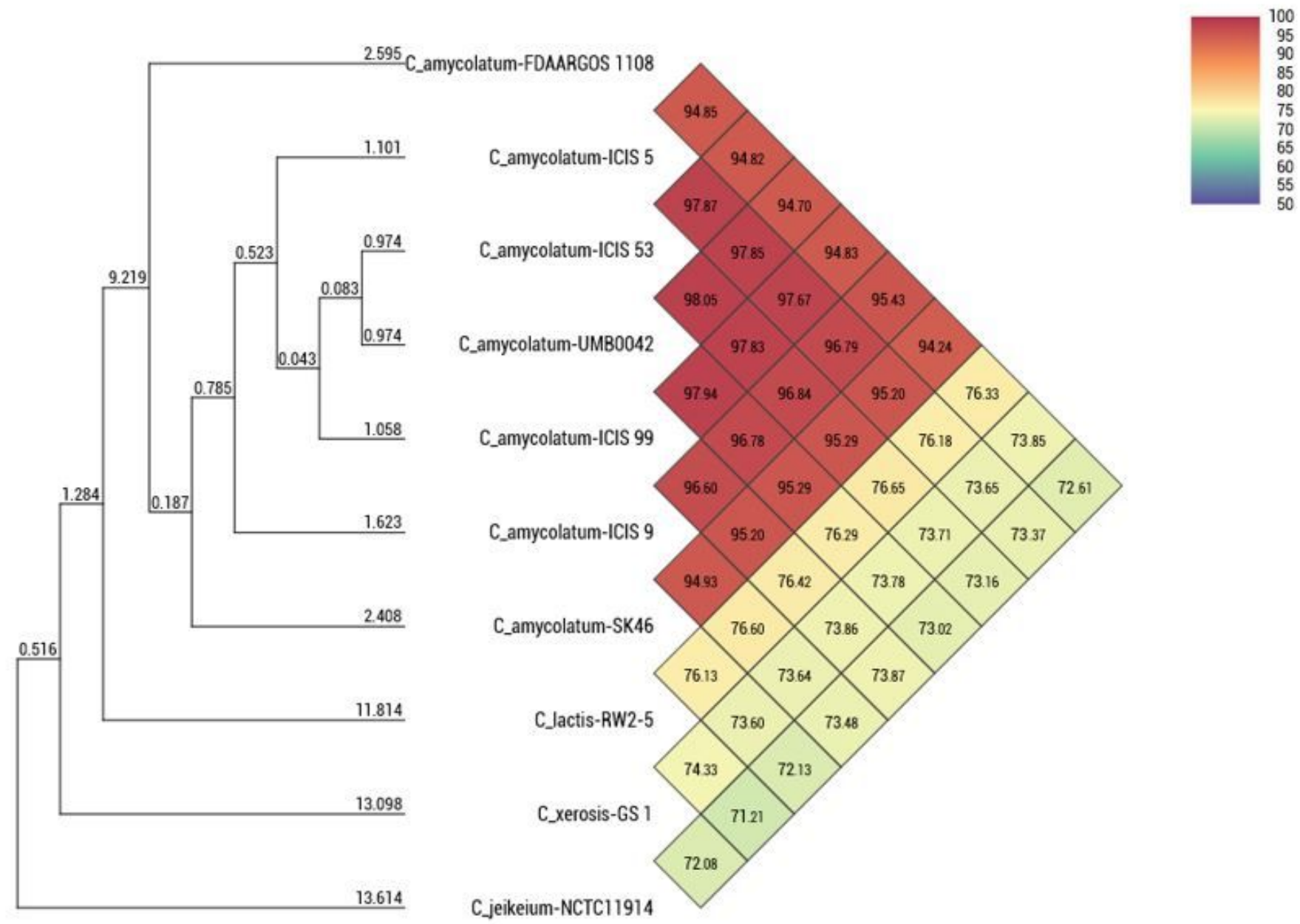

Figure 4

Heatmap generated with Original ANI values calculated from the OAT software.

Gene names

Predicted promoters

Predicted terminators

Show or hide small ORFS $\square$ No function determined

Blast hit with UniRef90

$\square$ Core Peptide

$\square$ Modification

$\square$ Immunity/Transport

$\square$ Regulation

$\square$ Transport \& Leader cleavage

Protease

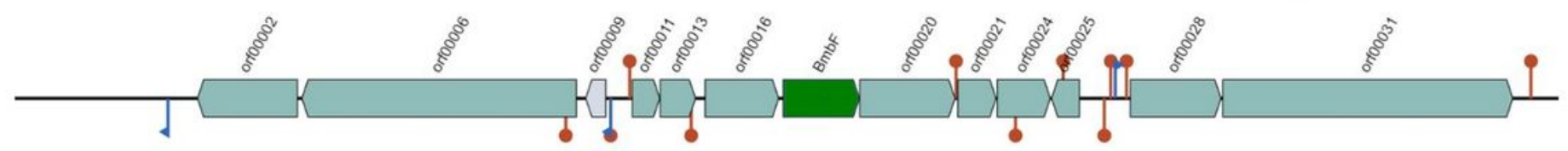


Figure 5

Sactipeptide encoded by genes represented by orf00016 predicted in ICIS 99 genome with BAGEL4.

\section{Supplementary Files}

This is a list of supplementary files associated with this preprint. Click to download.

- Supplementaryfails.docx 